\title{
Absorption spectroscopy of glucose based on a silicon photonics evanescent sensor
}

\author{
E. Ryckeboer, R. Bockstaele and R. Baets \\ Department of Information Technology, Photonics Research Group, Ghent University - imec \\ Center for Nano- and Biophotonics (NB-Photonics), Ghent University \\ Sint-Pietersnieuwstraat 41 B-9000 Ghent, Belgium \\ email: eva.ryckeboer@intec.ugent.be
}

\begin{abstract}
We present a silicon photonics evanescent sensor for glucose absorption spectroscopy. The important design aspects of this miniature sensor are discussed as well as the experimental challenges. We demonstrate detection of glucose down to 14 $\mathrm{mmol} / \mathrm{L}$, close to the physiological range of blood glucose in humans.

Index Terms-Silicon photonics, Biosensors, Spectroscopy
\end{abstract}

\section{INTRODUCTION}

For decades many research efforts have been geared towards the challenging development of a continuous glucose monitor [1]. The research is motivated by the strong increase in the number of patients with diabetes mellitus who can all benefit from tight blood glucose control. Key parameters for a glucose sensor are specificity, sensitivity and long-term stability. Whereas specificity and high sensitivity have been demonstrated by numerous sensor prototypes, long-term stability is yet to be fully mastered. We work towards an implantable device based on evanescent absorption spectroscopy in the near infrared. The specificity for glucose is determined by the uniqueness of its absorption spectrum. With this measurement principle, the use of reagents is avoided and multiple biomolecules apart from glucose can be detected in the same measurement. The glucose absorption spectrum has clear features in the first overtone band (1530-1820 nm), originating from the $\mathrm{C}-\mathrm{H}$ stretching vibrations. The sensor is an integrated photonic chip, fabricated in the high-index silicon-on-insulator (SOI) platform [2]. The small sensor footprint allows the device to be implanted. The main sensing element is a waveguide spiral whose evanescent field is attenuated according to the absorption spectrum of its environment. This work presents the first experimental results obtained with the evanescent waveguide spirals for the detection of glucose and discusses the challenges involved.

\section{Measurement PRINCIPLE}

To detect glucose, the transmitted power of the spiral waveguide, with a given path length $\mathrm{L}$, immersed in a glucose solution is measured. By using the Beer-Lambert law, this transmission depends on the glucose concentration $C_{\text {gluc }}$, taking into account the water displacement, as follows [3]:

$$
T=10^{-\left(\Gamma A_{s}+A_{w g}\right) \cdot L}
$$

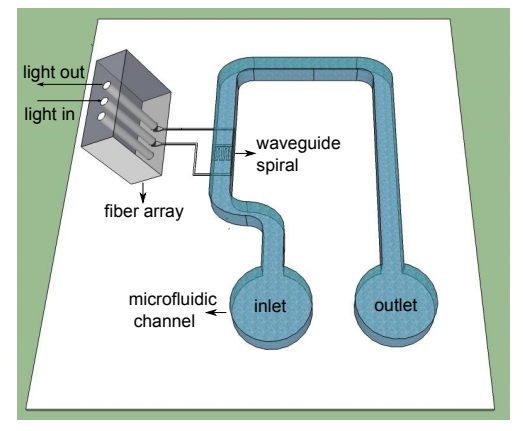

Fig. 1. Schematic of the sensor chip.

$$
A_{s}=\epsilon_{g l u c} C_{g l u c}+\epsilon_{\mathrm{H}_{2} \mathrm{O}}\left(C_{\mathrm{H}_{2} \mathrm{O}}-f C_{g l u c}\right)
$$

The absorbance due to waveguide losses is denoted with $A_{w g}$. The sample absorbance $A_{s}$ is multiplied with the confinement factor $\Gamma$, which is the fraction of the optical mode that extends into the cladding. The terms $\epsilon_{\text {gluc }}$ and $\epsilon_{\mathrm{H}_{2} \mathrm{O}}$ are the wavelength-dependent molar absorptivity of glucose and water respectively and define the shape of the spectrum. Given that the glucose molecules displace a number of water molecules from the optical path, the pure water concentration $C_{\mathrm{H}_{2} \mathrm{O}}$ is reduced with the glucose concentration by the displacement factor $\mathrm{f}$. The variation of glucose in physiological concentrations $(4-9 \mathrm{mmol} / \mathrm{L})$ alter the transmission with only a factor of 0.99971 to 0.99934 for a typical spiral length of $1 \mathrm{~cm}$ and confinement factor of $10 \%$. Therefore, a very high signal-tonoise set-up is required. The interaction length $\mathrm{L}$ is a design parameter that can be optimized for a given fill factor and waveguide losses. The goal is to optimize the dependence of detected power with glucose concentration. The optimum is obtained when the output power is a factor 1/e down from the input power and is therefore given by:

$$
L_{o p t}=\frac{1}{\Gamma \ln (10) A_{s}+\ln (10) A_{w g}}
$$

It is important to note that the optimum interaction length is wavelength dependent. 


\section{SENSOR DESIGN AND EXPERIMENTAL SET-UP}

The sensor layout is shown in figure 1. The chip has a $220 \mathrm{~nm}$ thick silicon layer on top of a $2 \mu \mathrm{m}$ thick buried oxide layer. The spiral is a $1 \mathrm{~cm}$ long rib waveguide with a $150 \mathrm{~nm}$ etch while the other waveguides are photonic wires with a $220 \mathrm{~nm}$ etch [4]. The choice for rib waveguide in the sensing element is evident from the optimum spiral length expression. A larger fill factor in combination with low loss waveguides is advantageous. The optical chip is combined with micro-fluidics in poly(dimethylsiloxane) (PDMS) using direct bonding. A micro-fluidics pump generates a steady flow in the $780 \mu \mathrm{m}$ wide and $50 \mu \mathrm{m}$ high microfluidic channel. Light is coupled in and out of the chip with a fiber array through grating couplers designed for TE light with a center wavelength of $1590 \mathrm{~nm}$, being the absorption peak wavelength of glucose. The fiber array is glued to the optical chip for mechanical stability. In addition, a temperature controller is used for thermal stability. The signal-to-noise ratio of the setup is dependent on the optical power of the source. When using higher power, the glucose detection limit will be lower. A tunable laser source is thus a tempting choice. Still, the coherent nature of a laser source in combination with relatively strong spurious reflections on the chip [5] gives rise to a noise-like ripple in the spectrum due to interference effects. Furthermore, this ripple exhibits time dependence due to temperature variation as well as vibrations and hence leads to real noise. Therefore, we opt for a broadband light source in combination with a tunable optical filter.

\section{EXPERIMENTAL RESULTS}

In a first experiment, we obtain the transmission spectrum of the spiral waveguide with and without glucose, by using an SLED in combination with a 2-nm bandpass filter that is tuned with a $0.5 \mathrm{~nm}$ step. First, the spectrum is measured during two consecutive wavelength-sweeps when pure DI water is applied to the micro-fluidic channel. The ratio of both measurements is shown in 2(a) and provides insight in the reproducibility of the measurement. Secondly, a glucose concentration of $277 \mathrm{mmol} / \mathrm{L}$ is applied by means of a manual valve. The ratio $P_{\text {glucose }} / P_{\text {water }}$ of the transmission spectrum with and without glucose can thus be obtained. The result is plotted in figure 2(a). We can conclude from this graph that a clear absorption dip is visible. In a second experiment, we monitored the transmitted power $\mathrm{P}(\mathrm{t})$ at a wavelength of $1590 \mathrm{~nm}$ of the spiral while switching three times between water and a glucose concentration of $14 \mathrm{mmol} / \mathrm{L}$, close to the physiological level. The result, after removing the envelope $\bar{E}$ due to slow drift, is plotted in figure 2(b). The inset shows the raw data of the evolution in transmitted power $\mathrm{P}(\mathrm{t}) / \mathrm{P}\left(t_{0}\right)$. Every time we switch to the glucose solution, a drop in transmission is detected. Additional measurements showed that the slow drift is wavelength dependent. This means that the measured glucose spectrum will be distorted when the acquisition time is slow. The main source of drift is attributed to polarization changes in the optical fibers. Future experiments will focus on dual-beam measurements with a faster acquisition rate and

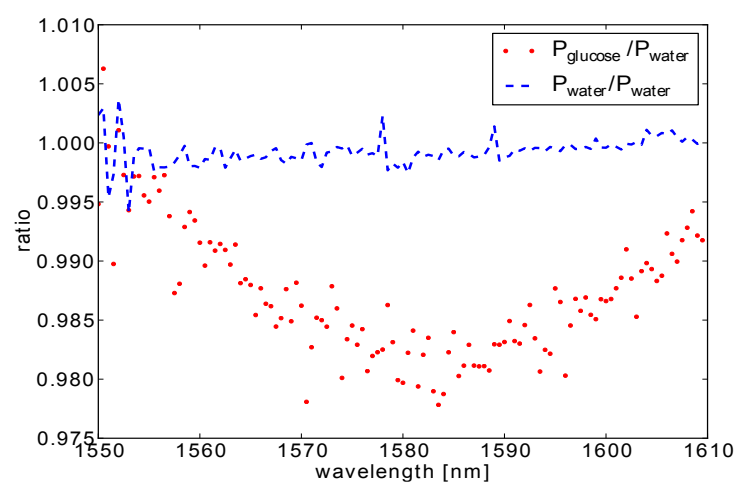

(a)

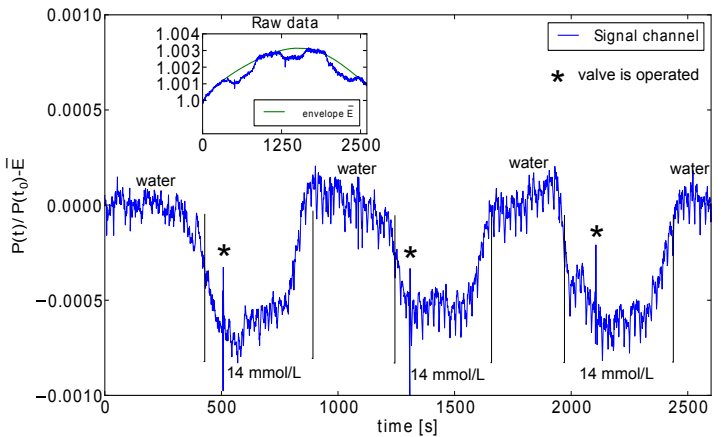

(b)

Fig. 2. (a) Ratio of consecutive transmission spectra. Blue curve for water/water and red curve for glucose $(227 \mathrm{mmol} / \mathrm{L}) /$ water (b) Change in transmission $\mathrm{P}(\mathrm{t}) / \mathrm{P}\left(t_{0}\right)$ at a wavelength of $1590 \mathrm{~nm}$, when switching between pure water and a $14 \mathrm{mMol}$ glucose solution after removing the envelope $\bar{E}$ caused by slow drift as shown in the inset.

an automated fluid switching. Working with a dual-beam setup will allow us to eliminate the slow drift effects as well as other common sources of noise. Multivariate analysis will also be used to measure glucose in mixtures with interfering biological molecules such as urea and lactate.

\section{Conclusions}

We present the first experimental demonstration of a singlebeam evanescent glucose sensor based on absorption spectroscopy. The important aspects of the sensor design are discussed as well as the experimental set-up. The measurement results include detection of glucose down to $14 \mathrm{mmol} / \mathrm{L}$ and indicate that the limit of detection is not yet reached. This provides a positive prospect for a continuous glucose monitoring device based on near-infrared absorption spectroscopy.

\section{ACKNOWLEDGEMENTS}

The authors acknowledge support from the IWT-SBO Glucosens project and from the ERC-InSpectra Advanced Grant.

\section{REFERENCES}

[1] S.Vaddiraju et al., J. Diabetes Sci. Technol.,4(6), p.1540 (2010)

[2] http://www.epixfab.eu/.

[3] A. Amerov et al., Appl. Spectrosc., 58(10), p1195-1204 (2004)

[4] S. Selvaraja et al.,"Record Low-Loss Hybrid Rib/Wire Waveguides for Silicon Photonic Circuits," Group IV Photonics (2010)

[5] F. Morichetti et al., Phys. Rev. Lett., 104(3), p.33902 (2010) 